\title{
RESEÑA DEL LIBRO RESPUESTAS AL TRANSHUMANISMO. CUERPO, AUTENTICIDAD Y SENTIDO DE SARA LUMBRERAS SANCHO (2020). DIGITAL REASON
}

\author{
Jonathan Piedra-Alegría \\ jonathan.piedra.alegria@una.cr \\ Universidad Nacional, Costa Rica
}

$\checkmark$ 1 libro de Sara Lumbreras Sancho, Respuestas al transhumanismo. Cuerpo, autenticidad y sentido (2020) presenta una amplia crítica sobre el transhumanismo (TH). Según su autora, el libro busca estimular el debate sobre algunos planteamientos transhumanistas. Para esto, realiza un examen de algunas de las principales propuestas de este movimiento a partir del concepto de human enhancement (mejora humana). El TH es una corriente cultural e intelectual que ha tomado fuerza en las últimas décadas. Pasó de una ideología de moda (en tanto conjunto de ideas) de un pequeño grupo de entusiastas de Silicon Valley a ser el pensamiento en boga entre médicos (Aubrey de Gray), ingenieros (Ray Kurweil) o exitosos empresarios automotrices (Elon Musk), por mencionar algunos ejemplos.

Desde los ámbitos académicos, las discusiones sobre el $\mathrm{TH}$, en forma usual, se dividen en dos campos opuestos: Los partidarios y los detractores (en ocasiones conocidos como bioconservadores). Es bastante habitual, también, que tanto uno como el otro den lecturas bastante parcializadas de los argumentos de los demás y es casi lo normal encontrar que ambos se mantienen imperturbables en sus propios campos, al ignorar las críticas o los puntos más moderados. Sin embargo, hay varios aspectos comunes entre los bioconservadores y los transhumanistas. En muchos casos, los dos grupos están de acuerdo en que nos enfrentamos a la posibilidad concreta de que tecnología pueda utilizarse para transformar la condición humana. También coinciden en que esto genera un compromiso ético sobre las implicaciones 
prácticas-individuales y sociales de su uso e implementación. Esta inquietud también recorre el libro de Lumbreras. Aunque con algunas diferencias. En primer lugar, a lo largo del texto nos encontramos con lo que podríamos llamar una propuesta crítica moderada. La obra pone en cuestión varios de los principales temas del TH: singularidad, la mejora corporal y cognitiva o la beneficencia procreativa. En este sentido, la obra cuestiona algunos de los core arguments que sostienen estas ideas. Desde un enfoque concreto, la autora realiza una selección de autores muy mediáticos (Elon Musk, Ray Kurzweil o Yuval Noah Harari) para realizar objeciones a muchas de sus tesis. A pesar de no ser un libro sobre ética, el estudio realizado también presenta esta dimensión, en particular con lo relacionado al uso de las tecnologías de mejora y, sobre todo, a las implicaciones del reduccionismo científico y el mecanicismo (subyacente en el TH) en las esferas moral y en la que la autora denomina como espiritual. Desde nuestro punto de vista estas serían las "Respuestas al transhumanismo" que le dan nombre al título de la obra. ¿La mejora tecnológica es una alternativa razonable para mejorar nuestro desempeño? ¿El cuerpo es importante a la hora de referirnos a la identidad? ¿El TH es una nueva forma de dualismo? ¿Cómo se entiende el concepto de autenticidad en los seres humanos? Todas estas son preguntas que obtendrán una respuesta durante el desarrollo del texto. Sin embargo, es claro que el planteamiento de ellas muestra la complejidad de los problemas y la dificultad para abordar muchos de los temas que plantea el TH. Con tal de resolver estas cuestiones, Lumbreras aborda estos tópicos de manera transversal mediante el concepto de autenticidad. Noción que, junto a la idea de subjetividad, serán centrales para el desarrollo de las críticas que plantea a lo largo del texto. Después de todo, ¿Cómo podríamos hablar de la autenticidad si no es por medio de la experiencia subjetiva? El problema de la experiencia subjetiva fue abarcado hace mucho tiempo por la Filosofía de la psicología cuando introducían el dilema relacionado con el método introspeccionista de Wundt. Es posible que las críticas a este método sean igual de válidas para la propuesta central del libro. Sin embargo, la autora tiene presente esto y aclara que, en este sentido, no busca la típica objetividad científica que, según ella, muchas veces responde a criterios materialistas y de mercado (capitalista).

De esta forma, el texto oscila entre una crítica argumentativa y un desarrollo original. Este sería un segundo punto por destacar: la propuesta 
de Lumbreras. Si bien ella no lo indica de manera directa, parece adscribirse a la teoría conocida como Embodied Cognition, al menos en ciertos puntos. Además, en la tercera parte del libro se realiza una interesante relación sobre el eje cerebro-intestino, que muestra cómo el estómago se comunica con el cerebro por el nervio vago, esto con tal de señalar la importancia del intestino para las actividades cognitivas. A pesar de que la tesis que desarrolla no es cognitivista, se utilizan algunas ideas relacionadas para fundamentar su propia propuesta: una espiritualidad encarnada. Este sería la aportación principal de la obra. Para esto, brinda un recorrido teórico y valorativo desde el tema de la trascendencia humana y el concepto de autenticidad. Grosso modo, diremos que esta es la parte más controvertida del libro, aspecto que no le resta mérito ya que su aporte radica en introducir al debate sobre la mejora humana, temas propios de los saberes ancestrales o prácticas no validadas por la tradicional episteme científica. En este punto la lectura se torna más atrayente por lo poco tradicional de su enfoque.

En términos generales, la obra discute aspectos que indagan el sentido del ser humano y la tecnología en un mundo en el que la inteligencia artificial o el deseo de vivir para siempre parecen ser temas cada vez más relevantes. Sería un error suponer que la autora critica sin más al $\mathrm{TH}$, al tomar como partida los elementos más caricaturescos y alocados del movimiento. En realidad, se introduce de manera seria en varios tópicos, con tal de brindar una alternativa que nos puede gustar o no, pero que toma en cuenta los avances científicos y tecnológicos. De esta forma, el texto concluye con una invitación a repensar estas cuestiones, que al final de cuentas es lo más importante. 